\title{
Cluster-randomized, controlled I2-month trial to evaluate the effect of a parental psychoeducation program on medication persistence in children with attention-deficit/hyperactivity disorder
}

This article was published in the following Dove Press journal:

Neuropsychiatric Disease and Treatment

13 June 2014

Number of times this article has been viewed

\author{
Alonso Montoya' \\ Amaia Hervás ${ }^{2}$ \\ Joaquín Fuentes ${ }^{3}$ \\ Esther Cardo ${ }^{4}$ \\ Pepa Polavieja ${ }^{5}$ \\ Javier Quintero6 \\ Rosemary Tannock ${ }^{7}$
}

'Medical Neurosciences, Lilly Research Laboratories Canada, Toronto, ON,

Canada; ${ }^{2} \mathrm{Child}$ and Adolescent Mental

Health Unit, Hospital Universitari

Mutua de Terrassa, and Developmental

Disorders Unit, Hospital Sant Joan de

Déu, Barcelona, ${ }^{3} \mathrm{Child}$ and Adolescent

Psychiatry Unit, Policlinica Gipuzkoa,

San Sebastian, ${ }^{4}$ Neuropediatric Unit,

Hospital Son Llatzer, University

of the Balearic Islands, Palma de

Mallorca, ${ }^{5}$ Department of Clinical

Research, Lilly Research Laboratories

Spain, Alcobendas, ${ }^{6}$ Department of

Psychiatry, Hospital Universitario

Infanta Leonor, Madrid, Spain; ${ }^{7}$ Applied

Psychology and Human Development, and Neurosciences and Mental Health Research Program, The Hospital for Sick Children, University of Toronto, Toronto, ON, Canada

Correspondence:Alonso Montoya Medical Neurosciences, Lilly Research Laboratories, Eli Lilly Canada Inc, 3650 Danforth Avenue, Toronto, ON, Canada

$\mathrm{Tel}+\mathrm{I} 4166933405$

Fax + I 4166997352

Email montoya_alonso@lilly.com
Background: This multicenter, cluster-randomized, nonblinded study evaluated the effect of parental psychoeducation on medication persistence among children and adolescents with newly diagnosed attention-deficit/hyperactivity disorder (ADHD).

Methods: Patients received standard medication alone or medication plus a parental psychoeducation program, and were followed for 12 months. The primary endpoint was time to withdrawal or termination of medication due to any cause. Secondary endpoints included change in ADHD symptom severity, functional outcome, program satisfaction, and safety.

Results: A total of 208 patients completed the study, which was terminated early because recruitment had ceased. At 12 months, there was no significant difference between the psychoeducation and control groups in the proportion of patients who discontinued pharmacologic treatment $(13.2 \%$ versus $14.3 \%$, respectively; size effect $-0.3, P=0.34$; hazard ratio 0.72 , $95 \%$ confidence interval $0.36-1.43$ ). Psychoeducation was associated with a significantly greater improvement in ADHD symptoms but not in functional outcome. Parental satisfaction with psychoeducation was high, and satisfaction with pharmacologic treatment was significantly greater in the psychoeducation group. There were no safety concerns.

Conclusion: No significant advantage for parental psychoeducation plus medication over medication alone in terms of time to medication withdrawal was observed. Psychoeducation had inconsistent but interesting effects on other outcomes.

Keywords: psychoeducation, medication persistence, attention-deficit/hyperactivity disorder, adjunctive psychoeducation, parents

\section{Introduction}

Attention-deficit/hyperactivity disorder (ADHD) is a neurodevelopmental disorder characterized by inappropriate inattention, hyperactivity, and impulsivity, ${ }^{1}$ and is estimated to occur in about $6.5 \%$ of school-aged children and $2.5 \%$ of adolescents. ${ }^{2}$ ADHD usually manifests during childhood and is associated with poor self-esteem, reduced social functioning, ${ }^{3}$ and an increased risk for long-term morbidity, including antisocial, mood, anxiety and/or addictive disorders, ${ }^{4}$ and mortality. ${ }^{5}$ Sufferers may also present with symptoms such as nocturnal enuresis ${ }^{6}$ and altered sleep patterns, ${ }^{7}$ which are commonly associated with sleep disorders (eg, sleep-related breathing disorder and obstructive sleep apnea syndrome $)^{8}$ and borderline intellectual functioning. ${ }^{9}$ 
The management of ADHD involves a multimodal approach that includes pharmacologic treatment, psychologic therapies, and/or psychosocial interventions. ${ }^{10-13}$ It should also involve the patient, parents, wider family, and school. Practice guidelines recommend psychoeducation as an important and front-line component of this multimodal approach. ${ }^{10,12,13}$ However, although it has been shown to have a generally beneficial impact on outcome, the available supporting evidence for the role of psychoeducation is limited and some findings are difficult to interpret. ${ }^{14}$

The benefit of pharmacologic intervention in patients with ADHD is well established. ${ }^{12,13}$ However, a significant proportion of parents (around one third) may refuse this option. ${ }^{15}$ In addition, adherence (the extent to which a patient acts in accordance with the prescribed dosing regimen, such as dose, timing, and frequency; also known as compliance) and persistence (the extent to which a patient continues to use a prescribed therapy over time) ${ }^{16}$ with ADHD medication are known to be problematic, ${ }^{17-19}$ and follow-up care after initiation of pharmacologic treatment can be poor. ${ }^{20}$ Although the degree of adherence to and persistence with medication appears to be dependent on the nature of the formulation and the drug, ${ }^{17-19}$ improving adherence to and persistence with ADHD treatment remains an important objective.

Parents in particular play a pivotal role in the decisionmaking process concerning the management of children with ADHD, ${ }^{21}$ and both their attitudes and their knowledge about ADHD may impact on adherence. ${ }^{22,23}$ For example, the results of a recent study suggest that provision of psychoeducation about ADHD to parents may improve outcomes, including adherence to prescribed pharmacologic treatments. ${ }^{24}$ However, despite being recommended by clinical practice guidelines, psychoeducational programs are rarely included in the treatment plan. ${ }^{25}$ Moreover, there is a lack of randomized controlled trials directly assessing the effects of psychoeducation on clinical outcome variables and on adherence and persistence in particular.

Against this background, we conducted a cluster-randomized study to evaluate the role of a structured parental psychoeducation program on medication persistence in the treatment of patients with ADHD. A cluster-randomized design was chosen for this investigation because the intervention was directed at groups or clusters (rather than individuals), and this design helps to minimize or remove potential contamination (eg, spread of the effect of an intervention to controls) between randomized groups. ${ }^{26}$ In addition, the participating institutions were carefully chosen in order to reflect the "real-world" situation, and not the type of environment encountered in specialized academic centers. We hypothesized that psychoeducation delivered to parents would improve medication persistence and hence overall treatment outcomes in children with ADHD.

\section{Materials and methods Study design}

This was a 12-month, multicenter, cluster-randomized, parallel-group, nonblinded trial of adjunctive parental psychoeducation plus medication versus medication alone on patient persistence with pharmacotherapy, involving the parents of patients aged 6-12 years with newly diagnosed ADHD. The psychoeducation program was designed specifically to be administered to small groups of parents (eg, 5-6 parents per group) within one month of receipt of a diagnosis of ADHD. ${ }^{27}$ It was a standardized, manual-based program that included materials for leaders (manual, slide deck) and for parents and children (eg, workbook, brochures, reading material), and was administered in five weekly 90-minute sessions (weeks 1-4 and week 9). The program was subjected to external peer review (ADHD clinical experts and advocacy groups) and a pilot test in a clinical environment before being included in this study.

Participating sites were required to have physicians with a medical specialty in psychiatry, child psychiatry, or child neurology, a potential recruitment rate of 3-5 patients per month, and not to have a structured psychoeducation program in place as part of routine care for ADHD patients. Centers recruited patients sequentially over time into clusters and each cluster was then randomly assigned, stratified by clinic size, to parental psychoeducation plus medication or to medication alone, according to a concealed computergenerated sequence provided by the sponsor. An interval of at least 4 weeks was mandated between a cluster starting treatment and recruitment of patients to a new cluster in order to avoid potential contamination (eg, sharing of information) by investigators and parents during the administration of simultaneous programs.

Following baseline assessments and randomization, patients commenced treatment with a standard course of medication alone or a standard course of medication in addition to parental psychoeducation. Medication was administered at the discretion of the attending physician in accordance with the ADHD guidelines produced by the National Institute for Health and Care Excellence. ${ }^{28}$ Parental psychoeducation sessions lasted for 90 minutes and were given once weekly for the first 4 weeks followed by a fifth session after a 5-week break. Sessions were offered at 
flexible times, although most took place in the late afternoon. They consisted of lectures, small-group and large-group discussions, shared learning from previous sessions, and homework. Details of session content are provided in Table 1 and include provision of information on ADHD in general, pharmacologic management, and behavior management. Patients and parents attended the clinic for up to 12 months of follow-up, with assessments at baseline and at weeks 4, 12,24 , and 52 following randomization. In addition, patient progress was monitored by telephone call between each clinic visit (a total of four telephone calls).

The trial was conducted in 27 centers in Spain. It adhered to the principles of the Declaration of Helsinki and was approved by local ethical review boards. All parents provided written informed consent to participation in the trial and randomization to medication or to medication plus psychoeducation.

\section{Patients and parents}

Eligible patients were children or adolescents aged 6-12 years with a clinically confirmed diagnosis of ADHD (Diagnostic and Statistical Manual of Mental Disorders, Text Revision Fourth Edition [DSM-IV-TR] criteria), an Attention Deficit Hyperactivity Disorder Rating Scale
IV-Parent Version (ADHD-RS-IV Parent:Inv) score at least 1.5 standard deviations above the age norm for their diagnostic subtype, and a Clinical Global ImpressionADHD Severity (CGI-ADHD-S) score $\geq 4$ at baseline. Patients were required to be pharmacologically naïve and willing to commence on medication at the same time as the first planned psychoeducation session. Adjustment of doses of pharmacologic treatment was allowed at the discretion of the prescribing physician. The presence of any learning difficulties, based on patient medical history and physician reports, was recorded.

Participating parents/guardians were required to be the primary caregiver and legal guardian of the patient at the time of initial diagnosis of ADHD. Before randomization, parents/guardians were also required to agree to possible participation in the psychoeducation program. Parents/ guardians were not eligible for inclusion if pharmacologic treatment for ADHD was contraindicated for their children, or if either the parent/guardian or child was likely to start a structured psychoeducation program for ADHD outside of this trial. Parents/guardians were also excluded if their children had a history of bipolar disorder, psychosis, or autism spectrum disorder, or were in any way unsuitable to participate in the study.

Table I Content of the psychoeducation sessions

\begin{tabular}{ll}
\hline Session & Description \\
\hline$I^{\text {a }}$ & Overview of program content and objectives \\
& ADHD etiology, epidemiology, symptomatology, comorbidities, diagnosis, treatment, prognosis \\
& Reasons and sources of bad behavior in children with ADHD and influence on family interactions \\
& Situational circumstances \\
& Techniques oriented to improve adequate behavior \\
& Time optimization \\
& Reinforcements (verbal, social, physical) \\
& Positive care \\
& Token system \\
& Techniques oriented to diminish inadequate behavior \\
& How to give commands efficiently \\
& Outdoor time \\
& How to handle inadequate behavior in public places \\
& Social skills \\
& Emotional recognition and expression \\
& Training in social problem-solving \\
& Variables influencing attention problems \\
& Techniques to improve attention problems \\
& Interventions for attention problems \\
& How to support homework \\
& Barkley's decalog \\
& Recap of previous four sessions \\
\hline
\end{tabular}

Notes: ${ }^{\mathrm{A} A f t e r}$ the first and subsequent three sessions, participants were encouraged to apply what they had learned and to discuss that in the next session. ${ }^{b}$ After session 3 , parents completed a form describing the child's and parent's temperaments, and the existence of potential stressing factors in the family that may contribute to worsening of family dynamics.

Abbreviation: ADHD, attention-deficit/hyperactivity disorder. 


\section{Endpoints}

The primary endpoint of this study was the duration of time in days until withdrawal or termination of medication due to any cause during the 12-month study period. For patients who were lost to follow-up or who started ineligible psychotherapeutic (pharmacologic and nonpharmacologic) treatment during the study, time to withdrawal was censored at the date of the last assessment. Secondary endpoints included the change from baseline to weeks 4, 12, 24, and 52 in the ADHD-RS-IV Parent:Inv score. The ADHD-RS-IV Parent:Inv is an 18-item scale with one item for each of the 18 symptoms contained in the DSM-IV diagnosis of ADHD, each item being scored on a scale of $0-3(0$, never or rarely; 1 , sometimes; 2 , often; 3 , very often). The rating scale was used to assess symptom severity over the past week, and was administered and scored by qualified personnel at the investigative site based on an interview with the parent and the patient (not necessarily conducted at the same time). ${ }^{29}$ The total score was computed as the sum of the scores on each of the 18 items. Inattention and hyperactivity-impulsivity subscale scores were also computed.

The change in CGI-ADHD-S score from baseline to weeks $4,12,24$, and 52 was also evaluated. The CGIADHD-S $\mathrm{S}^{30}$ is a single-item clinician rating of the severity of ADHD symptoms in relation to the clinician's total experience of patients with ADHD. Severity is rated on a 7-point scale (1, normal, not ill at all; 7, among the most extremely ill patients).

The functional outcome of patients at week 52 was assessed using the Weiss Functional Impairment Rating Scale-Parent version (WFIRS-P). This is a parent-completed questionnaire that measures the impact of ADHD on their child's functioning in multiple domains. ${ }^{12,31}$ It rates impairment in six domains of functioning, ie, home, school, self-concept, social, activities of daily living, and risk taking. Each item is rated by the parent on a 4-point Likert scale of $0-3(0$, never or not at all; 1 , sometimes or somewhat; 2 , often or much; 3 , very often or very much). An average score was calculated by summing all items rated 0 through 3 and dividing the total by the number of nonmissing items. Items rated as not applicable were excluded from the calculation. Average scores were calculated for all six domains (total score) and for each subdomain.

Satisfaction with pharmacologic treatment at week 52 was assessed using the Treatment Satisfaction Questionnaire. This parent-administered questionnaire consists of five questions relating to treatment satisfaction that are each scored on a scale of $0-4$ ( 0 , very satisfied; 4 , very dissatisfied).
It assesses a parent's satisfaction with his or her child's pharmacologic treatment over the past weeks. ${ }^{32}$

Treatment switching throughout the 12-month study period, the impact of therapy on the reasons for discontinuation, and satisfaction with the psychoeducation program (evaluation of home activities, handouts, and the session leaders' preparation, teaching, interest, concern, and helpfulness) were also evaluated. Safety and tolerability were assessed by recording adverse events, their attribution to study procedures and interventions, and their severity and seriousness. In addition, adherence to pharmacologic treatment was evaluated at each visit by direct questioning of the parents.

\section{Statistical analysis}

Based on the primary endpoint of time to discontinuation of treatment (any cause) and assuming discontinuation rates of $32 \%$ for the psychoeducation group and $17 \%$ for the control group, ${ }^{33}$ it was estimated that a sample size of 360 patients would provide $80 \%$ power to detect hazard ratios of $\geq 2.07$ or $\leq 0.48$ at the 0.05 level of significance. In addition, a design effect correction was applied to the estimated number of events, ${ }^{34}$ that took into account the intracluster correlation. Assuming that approximately 90 clusters were randomized with an average number of four patients per cluster and an intracluster correlation of $0.05,{ }^{35}$ the design effect would be 1.2 and the corrected estimated number of events would be 75. This study required 90 completed patient clusters with an average of four patients per cluster. A completed patient cluster is defined as one with a minimum of three patients.

All analyses were conducted on the full analysis set, which consisted of all randomized clusters of patients who were diagnosed with ADHD. All analyses accounted for clustering and site effects, and all tests of treatment effects were conducted at a $5 \%$ two-sided alpha level of significance.

For the primary endpoint, the distribution of time to discontinuation of treatment was estimated using the KaplanMeier product-limit method..$^{36}$ The proportion of patients still receiving treatment at 6,10 , and 12 months, and the associated 95\% confidence intervals (CIs), were reported according to intervention group. Differences between the psychoeducation and control groups were estimated using a marginal Cox proportional hazards mode ${ }^{37}$ that accounted for clustering using a "robust sandwich variance" estimator. A basic model with the covariates of clinic size, type of ADHD diagnosis, and patient comorbidity was fitted to estimate and test for group differences.

Data for secondary endpoints (ADHD-RS-IV Parent:Inv, CGI-ADHD-S, and treatment satisfaction) followed a 
clustered longitudinal structure, in which individuals were nested within batches, and treatment was randomly assigned to batch and repeated measures (visits). These data were analyzed using a mixed model for repeated measures, which took into account the clustered structure of the data and allowed the residuals associated with longitudinal measures on the same individuals to be correlated. Batches and participants nested within each batch were assumed to be random factors. The model also included treatment, visit, interaction treatment by visit, and clinic size as fixed effects. An unstructured covariance matrix was assumed for the residuals. Intracluster correlation coefficients were estimated for all secondary endpoints using the mixed model for repeated measures.

Last observation carried forward change from baseline to endpoint analysis was conducted for the overall score and domains of the WFIRS-P, adjusting for clinic size and clustering. For analysis of the last observation carried forward change from baseline to endpoint, patients with a baseline value and at least one postbaseline measurement were included in the analysis. Analyses involved an extension of analysis of covariance, adjusting for the effect of clustering (ie, a mixed effects analysis of covariance with clusters nested in the intervention group). All statistical analyses were performed using SAS version 9.2 (SAS Institute, Cary, NC, USA).

\section{Results \\ Study termination}

This study was terminated early because recruitment had slowed dramatically despite extension of the recruitment period. The decision was made to analyze the available data even though the study would be underpowered, because prolonging recruitment for a longer period could put the validity of the data at risk due to a lack of control over evolving environmental conditions. Thus, compared with the 90 clusters and 360 patients required, only 65 clusters and 272 patients entered the study between May 2009 and October 2012.

\section{Baseline characteristics and patient disposition}

Patient characteristics were broadly similar at baseline for the two groups (Table 2). Mean age was approximately 9 years in both groups, and most patients were male $(>70 \%)$ and were living in a family environment with both biologic parents $(>79 \%)$, with the mother being recorded as the primary caregiver $(>86 \%)$. Most patients had received a diagnosis of combined (inattentive and impulsive) ADHD (>61\%), while mean baseline scores for ADHD-RS-IV Parent:Inv ranged from 36.2 to 39.5 and those for the WFIRS-P total score from 0.7 to 0.8 .

Patient disposition is shown in Figure 1. Of the 272 patients randomized to the 65 clusters (mean 4.2 patients per cluster), 270 entered the study and were included in the full analysis set; 208 patients completed the 12-month investigation period. A total of 28 patients discontinued the study in the psychoeducation group (19.4\%) compared with 34 patients in the control group (27.0\%), and the reasons for discontinuation were similar in the two groups (Table 3).

The most frequently prescribed ADHD agents at baseline and during the study were long-acting methylphenidate (Concerta ${ }^{\circledR}$ [Janssen Pharmaceuticals, Inc., Titusville, NJ, USA]; Medikinet ${ }^{\circledR}$ [Medice Arzneimittel Pütter $\mathrm{GmbH}$ \& Co. KG, Iserlohn, Germany]), atomoxetine (Strattera ${ }^{\circledR}$ [Lilly USA, LLC, Indianapolis, Indiana, USA]), and short-acting methylphenidate (Rubifen ${ }^{\circledR}$ [Laboratorios Rubió, Barcelona, Spain]; Figure 2). Use of drug type was broadly similar across the two groups, as was drug dosage.

\section{Program details}

Most of the program sessions (56\%) took place in the afternoon, 24\% took place in the morning, $12 \%$ took place at a time suitable for the participating families, and the remainder $(8 \%)$ took place at mixed times. Attendance at the psychoeducation program was $95.8 \%$ for session 1 (baseline), $88.2 \%$ for session $2,84.7 \%$ for session $3,82.6 \%$ for session 4 , and $73.6 \%$ for session 5 . Most cases of nonattendance at the psychoeducation program were due to study withdrawals. The parents of 43 patients (12 clusters) attended at least one program session during the school summer holidays (July and August). Most of them (30 parents) attended just one session.

\section{Primary endpoint}

At 12 months, 19 patients (13.2\%) in the psychoeducation group and 18 patients $(14.3 \%)$ in the control group had discontinued pharmacologic treatment (Figure 3). There was no significant difference between groups in time to discontinuation (size effect $-0.3 ; P=0.34$; hazard ratio $0.72,95 \% \mathrm{CI}$ $0.36-1.43)$. Time to discontinuation was largely unaffected by the presence or absence of comorbidity. However, patients with specific learning difficulties (diagnosis based on patient medical history and physician reports) were significantly more likely to discontinue $(P<0.001)$ than those without such problems (Table 4). 
Table 2 Baseline patient characteristics

\begin{tabular}{|c|c|c|}
\hline Characteristic & Psychoeducation group $(n=144)$ & Control group $(n=126)$ \\
\hline Age, years (mean [SD]) & $9.3(1.9)$ & $8.8(1.8)$ \\
\hline \multicolumn{3}{|l|}{ Sex, n (\%) } \\
\hline Female & $38(26.4)$ & $37(29.4)$ \\
\hline Male & $106(73.6)$ & $89(70.6)$ \\
\hline \multicolumn{3}{|l|}{ Family type, n (\%) } \\
\hline Nuclear family & $118(81.9)$ & $100(79.4)$ \\
\hline Adoptive parent & $9(6.3)$ & $10(7.9)$ \\
\hline Divorced parent & $16(11.1)$ & $12(9.5)$ \\
\hline Single parent & $\mathrm{I}(0.7)$ & $3(2.4)$ \\
\hline Other & $0(0)$ & $\mathrm{I}(0.8)$ \\
\hline \multicolumn{3}{|l|}{ Primary caregiver, n (\%) } \\
\hline Mother & $133(92.4)$ & $109(86.5)$ \\
\hline Father & II (7.6) & $16(12.7)$ \\
\hline Other & $0(0)$ & $\mathrm{I}(0.8)$ \\
\hline \multicolumn{3}{|l|}{ Disorder, n (\%) } \\
\hline Affective & $2(1.4)$ & $0(0)$ \\
\hline Anxiety & $4(2.8)$ & $8(6.3)$ \\
\hline Oppositional defiant & $19(13.2)$ & $12(9.5)$ \\
\hline Conduct & $7(4.9)$ & $5(4.0)$ \\
\hline Learning & $29(20.1)$ & $22(17.5)$ \\
\hline Time since diagnosis, days (mean [SD]) & $6.7(8.4)$ & $7.2(8.6)$ \\
\hline \multicolumn{3}{|l|}{ Diagnosis subtype, $\mathrm{n}(\%)$} \\
\hline Inattentive & $47(32.6)$ & $21(16.7)$ \\
\hline Hyperactive & $8(5.6)$ & $2(1.6)$ \\
\hline Combined & $89(6 I .8)$ & $103(8 \mid .7)$ \\
\hline ADHD-RS-IV Parent:Inv score, mean (SD) & $36.2(9.0)$ & $39.5(9.0)$ \\
\hline CGI-ADHD-S score, mean (SD) & $5.0(0.9)$ & $5.0(1.0)$ \\
\hline \multicolumn{3}{|l|}{ WFIRS-P score, mean (SD) } \\
\hline Total & $0.8(0.4)$ & $0.8(0.4)$ \\
\hline Home domain & $1.0(0.6)$ & $1.0(0.6)$ \\
\hline School domain & $\mathrm{I} .0(0.5)$ & $\mathrm{I} .0(0.5)$ \\
\hline Self-concept domain & $0.9(0.8)$ & $0.8(0.8)$ \\
\hline Social domain & $0.8(0.6)$ & $0.7(0.6)$ \\
\hline Activities of daily living domain & $0.9(0.5)$ & $0.9(0.4)$ \\
\hline Risk-taking domain & $0.4(0.4)$ & $0.4(0.4)$ \\
\hline
\end{tabular}

Abbreviations: ADHD-RS-IV Parent:Inv, Attention-Deficit/Hyperactivity Disorder Rating Scale IV-Parent version; CGI-ADHD-S, Clinical Global Impression ADHD-Severity scale; SD, standard deviation; WFIRS-P, Weiss Functional Impairment Rating Scale-Parent version.

\section{Secondary endpoints}

Intergroup comparison of the change from baseline in ADHD-RS-IV Parent:Inv total score gave an estimated adjusted mean (least square mean [LSM]) of $-3.362 ; 95 \%$ $\mathrm{CI}-6.335,-0.389 ; P=0.027)$ in favor of the psychoeducation group at 12 months (Figure 4). There was also a significant difference between the groups for the inattention subscore in favor of the psychoeducation cohort (LSM $-1.863 ; 95 \%$ CI $-3.480,-0.247 ; P=0.024)$ but not for the hyperactivity/ impulsivity subscore (LSM -1.498; 95\% CI -3.125, 0.128; $P=0.071$ ). The intracluster correlation coefficient for each score was $0.084,0.075$, and 0.089 , respectively.

The change from baseline in the CGI-ADHD-S score gave an LSM estimate of -0.385 , again in favor of psychoeducation compared with the control group (95\% CI $-0.773,0.002 ; P=0.051$, Figure 5).

There was no significant difference between the two groups in the WFIRS-P total score (LSM $-0.059 ; 95 \%$ CI $-0.134,0.017 ; P=0.13$ ), or when the subdomain scores were evaluated; home (LSM $-0.058 ; 95 \%$ CI -0.173 , $0.058 ; P=0.33$ ), school (LSM $-0.063 ; 95 \%$ CI -0.166 , $0.039 ; P=0.23$ ), self-concept (LSM $-0.101 ; 95 \% \mathrm{CI}-0.245$, $0.044 ; P=0.17$ ), social (LSM $-0.016 ; 95 \%$ CI $-0.110,0.078$; $P=0.74$ ), activities of daily living (LSM $-0.048 ; 95 \%$ CI $-0.138,0.042 ; P=0.29$ ), and risk-taking (LSM -0.013 ; 95\% CI -0.096, 0.070; $P=0.75$ ).

Parents' ratings of satisfaction with the psychoeducation program (canvassed after the fifth session) revealed that 


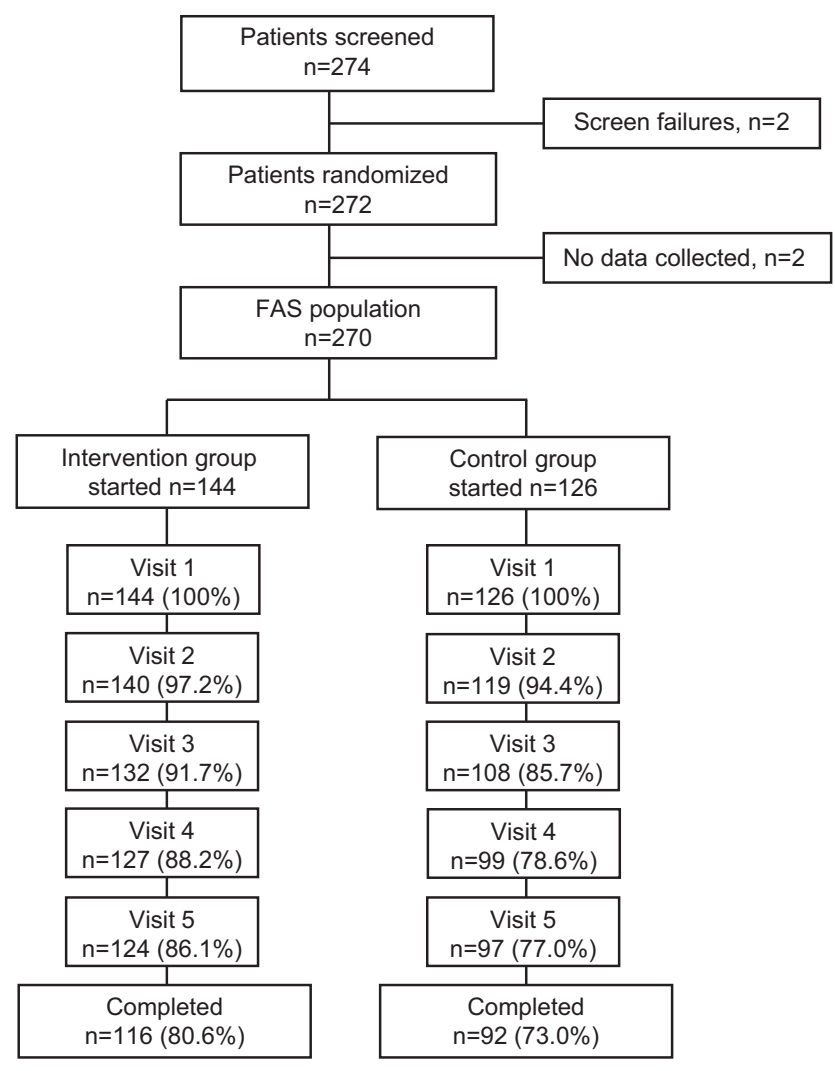

Figure I Patient disposition.

Abbreviation: FAS, full analysis set.

$90.3 \%$ found the home activities to be somewhat or extremely useful, and $84.2 \%$ of patients reported similar usefulness categories for the weekly handout materials. The session leaders' preparation and teaching was rated as being somewhat or very good by $98.1 \%$ and $99.0 \%$ of attendees, respectively. The same high proportion of parents $(99.0 \%)$ was either somewhat or very satisfied with the leaders' interest and concern, and $99.0 \%$ found the leader to be either somewhat or extremely helpful.

Treatment satisfaction with pharmacologic intervention was significantly greater in the psychoeducation group (LSM -0.417; 95\% CI $-0.712,-0.122 ; P=0.006$ ). Drug usage patterns, including treatment switches, additions, dose modifications, and treatment discontinuations, are presented in Figure 6. A higher proportion of patients in the psychoeducation group $(n=65,45.1 \%)$ than in the control group $(n=43$, $34.1 \%$ ) maintained their original drug and dosage throughout the 12 months of the study. Furthermore, nonadherence (at least one missed dose during the study) with pharmacologic treatment for ADHD was very low. Overall, two patients in the psychoeducation group (1.4\%) and six patients in the control group (4.8\%) were reported to be nonadherent at least once.

A total of 114 patients (42.2\%) reported at least one protocol-emergent adverse event and the number of events was similar for the two groups (Table 5). Adverse events leading to discontinuation were reported by two patients $(1.4 \%)$ in the psychoeducation group (dystonia and glaucoma) and seven patients (5.6\%) in the control group (irritability, malaise, pyrexia, tachycardia, akathisia, decreased appetite, and aggression). Serious adverse events were reported by three patients (2.1\%) in the psychoeducation group (pneumonia $n=2$; femur fracture $n=1$ ) and one patient $(0.8 \%)$ in the control group (appendicitis). All serious adverse events were considered to be unrelated to the study procedures and interventions or (probably) to ADHD medication.

Table 3 Discontinuations during the study

\begin{tabular}{|c|c|c|}
\hline Parameter & $\begin{array}{l}\text { Psychoeducation } \\
(n=\mid 44)\end{array}$ & $\begin{array}{l}\text { Control } \\
(n=126)\end{array}$ \\
\hline Completed (n [\%]) & $116(80.6)$ & $92(73.0)$ \\
\hline \multicolumn{3}{|l|}{ Reason for discontinuation ( $[\%]$ ) } \\
\hline Cluster dissolution & $0(0.0)$ & $2(1.6)$ \\
\hline Adverse event & $2(1.4)$ & $7(5.6)$ \\
\hline Death & $0(0.0)$ & $0(0.0)$ \\
\hline Protocol violation & $0(0.0)$ & $3(2.4)$ \\
\hline Subject decision & $\mathrm{I}(0.7)$ & $0(0.0)$ \\
\hline Physician decision & $0(0.0)$ & $3(2.4)$ \\
\hline Sponsor decision & $5(3.5)$ & $0(0.0)$ \\
\hline Parent/caregiver decision & $10(6.9)$ & $12(9.5)$ \\
\hline Start of structured psychotherapy aimed at ADHD & I (0.7) & $0(0.0)$ \\
\hline Pharmacologic withdrawal & I (0.7) & I (0.8) \\
\hline Lost to follow-up & $8(5.6)$ & $3(2.4)$ \\
\hline Unknown & $0(0.0)$ & $3(2.4)$ \\
\hline
\end{tabular}

Abbreviation: ADHD, attention-deficit/hyperactivity disorder. 


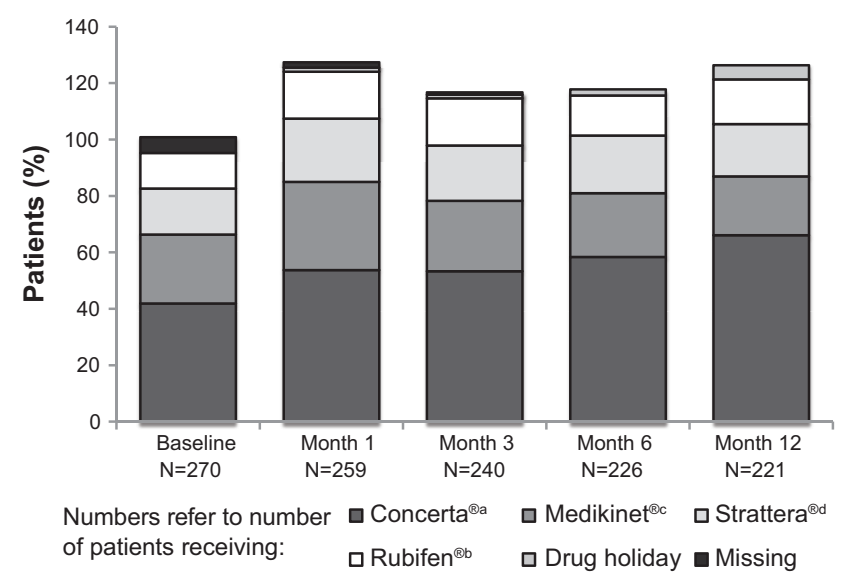

Figure 2 Pharmacologic treatment usage during the study.

Notes: ajanssen Pharmaceuticals, Inc., Titusville, NJ, USA; 'Laboratorios Rubió, Barcelona, Spain; 'Medice Arzneimittel Pütter GmbH \& Co. KG, Iserlohn, Germany; 'Lilly USA, LLC, Indianapolis, IN, USA.

\section{Discussion}

At the time of conceptualization of this study, there was no randomized controlled investigation assessing the impact of psychoeducation on clinical variables in patients who were newly diagnosed or naïve to treatment. Therefore, the present study was conducted to further define the role of psychoeducation in the treatment of ADHD by evaluating the effect of a structured parental psychoeducation program on clinical outcomes and, in particular, medication persistence among patients.

The psychoeducation program was a standardized, manual-based program designed for parents of patients with ADHD. The parent-orientated approach of the program reflects the pivotal role that parents play in the decision-making process for children with $\mathrm{ADHD}^{21}$ and the fact that their attitudes may impact on adherence. ${ }^{22-24}$

In this study, administration of a structured psychoeducation program to parents of patients with a new diagnosis of ADHD requiring medication did not significantly affect medication persistence in terms of time to treatment withdrawal after 12 months of pharmacotherapy (primary endpoint). However, it is also notable that only 62 patients withdrew for any reason during the study, and that the number of patients affected was similarly low in the psychoeducation $(n=28)$ and control $(n=34)$ groups. It is also interesting to note that nonadherence to ADHD pharmacologic treatment was very low in both groups (despite the fact that the control group did not undergo psychoeducation), with only two patients $(1.4 \%)$ in the psychoeducation group and six patients $(4.8 \%)$ in the control group being nonadherent at least once during the study. These figures are lower than the nonadherence rates of $7 \%-81 \%$ reported in recent publications, ${ }^{38-44}$ depending on the drug and formulation used. Of note, the presence of learning difficulties increased the likelihood of treatment discontinuation by approximately three-fold in our investigation. It has been suggested that learning difficulties have an impact on adherence to pharmacologic treatment and that ADHD patients with learning difficulties may have a poorer response to pharmacologic treatment compared with those without this comorbidity. ${ }^{45-47}$

A statistically significant difference in improvement of clinical symptoms as measured by the ADHD-RS-IV Parent:Inv and severity of clinical symptoms as measured by the CGI-ADHD-S was observed between the groups,

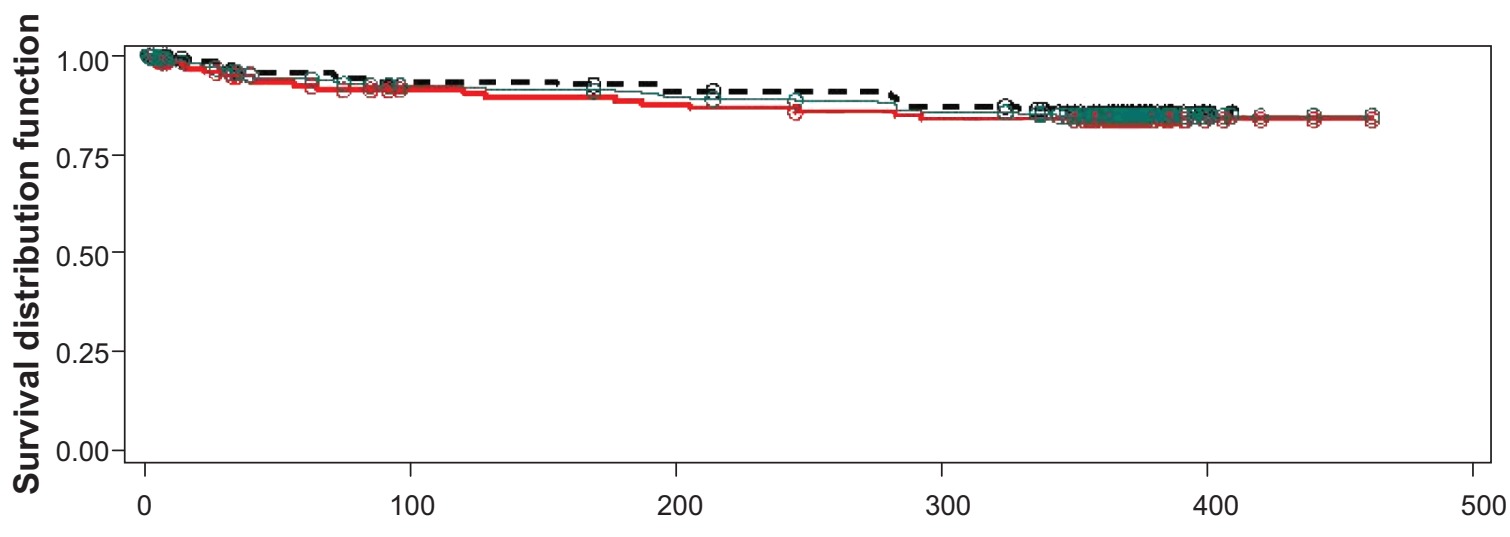

Time to discontinuation of pharmacological treatment (days)

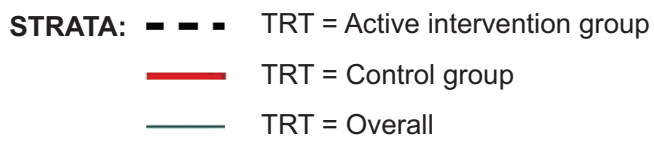

STRATA: - - - TRT = Active intervention group

TRT $=$ Control group

TRT $=$ Overall

O $D$ Censored TRT = Active intervention group

O O O Censored TRT = Control group

○ $\odot$ Censored TRT = Overall

Figure 3 Estimated time to treatment discontinuation (Kaplan-Meier). Abbreviation: TRT, treatment. 
Table 4 Cox proportional hazards model for time until treatment discontinuation $\leq 12$ months; analysis of covariates

\begin{tabular}{|c|c|c|c|c|c|}
\hline & \multirow{2}{*}{$\begin{array}{l}\text { Parameter } \\
\text { estimate }\end{array}$} & \multirow[t]{2}{*}{$P$-value } & \multirow[t]{2}{*}{ HR } & \multicolumn{2}{|l|}{$95 \% \mathrm{Cl}$} \\
\hline & & & & Lower & Upper \\
\hline Treatment (intervention or control) & -0.3 & 0.343 & 0.715 & 0.358 & 1.430 \\
\hline Type of diagnosis ${ }^{\mathrm{a}}$ & -0.0 & 0.911 & 0.956 & 0.430 & 2.123 \\
\hline \multicolumn{6}{|l|}{ Comorbidity $^{b}$} \\
\hline Oppositional defiant disorders & -0.0 & 0.931 & 0.956 & 0.349 & 2.624 \\
\hline Anxiety disorders & -0.2 & 0.807 & 0.832 & 0.191 & 3.628 \\
\hline Learning disorders & 1.2 & $<0.001$ & 3.189 & 1.654 & 6.146 \\
\hline Conduct disorders & 0.4 & 0.561 & 1.484 & 0.392 & 5.613 \\
\hline Affective disorders & 1.6 & 0.057 & 5.158 & 0.952 & 27.931 \\
\hline
\end{tabular}

Notes: aHyperactive or combined versus inattentive. bPresence versus absence.

Abbreviations: $\mathrm{Cl}$, confidence interval; $\mathrm{HR}$, hazard ratio.

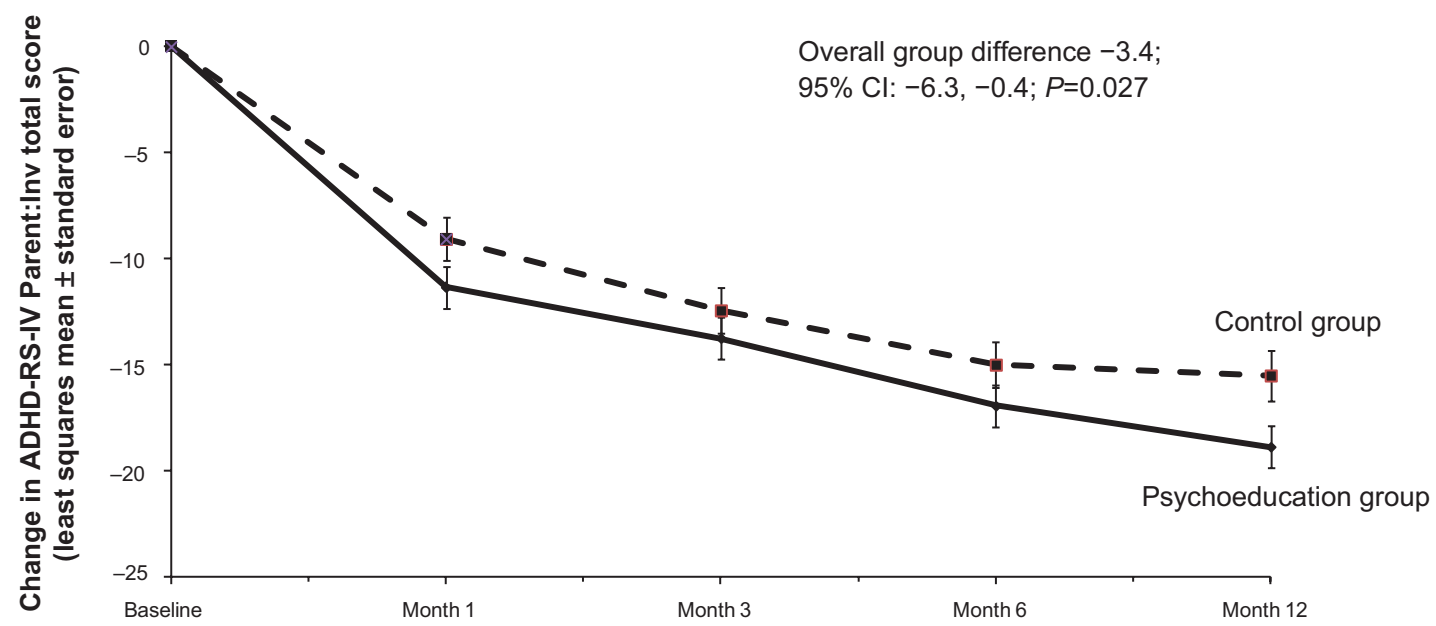

ADHD-RS-IV Parent:Inv total scale ranges from 0 (minimum) to 54 (maximum)

Figure 4 Change in ADHD-RS-IV Parent:Inv total score (least squares mean estimate) for the psychoeducation and control groups.

Abbreviations: ADHD-RS-IV Parent:Inv, Attention-Deficit/Hyperactivity Disorder Rating Scale IV-Parent version; Cl, confidence interval.

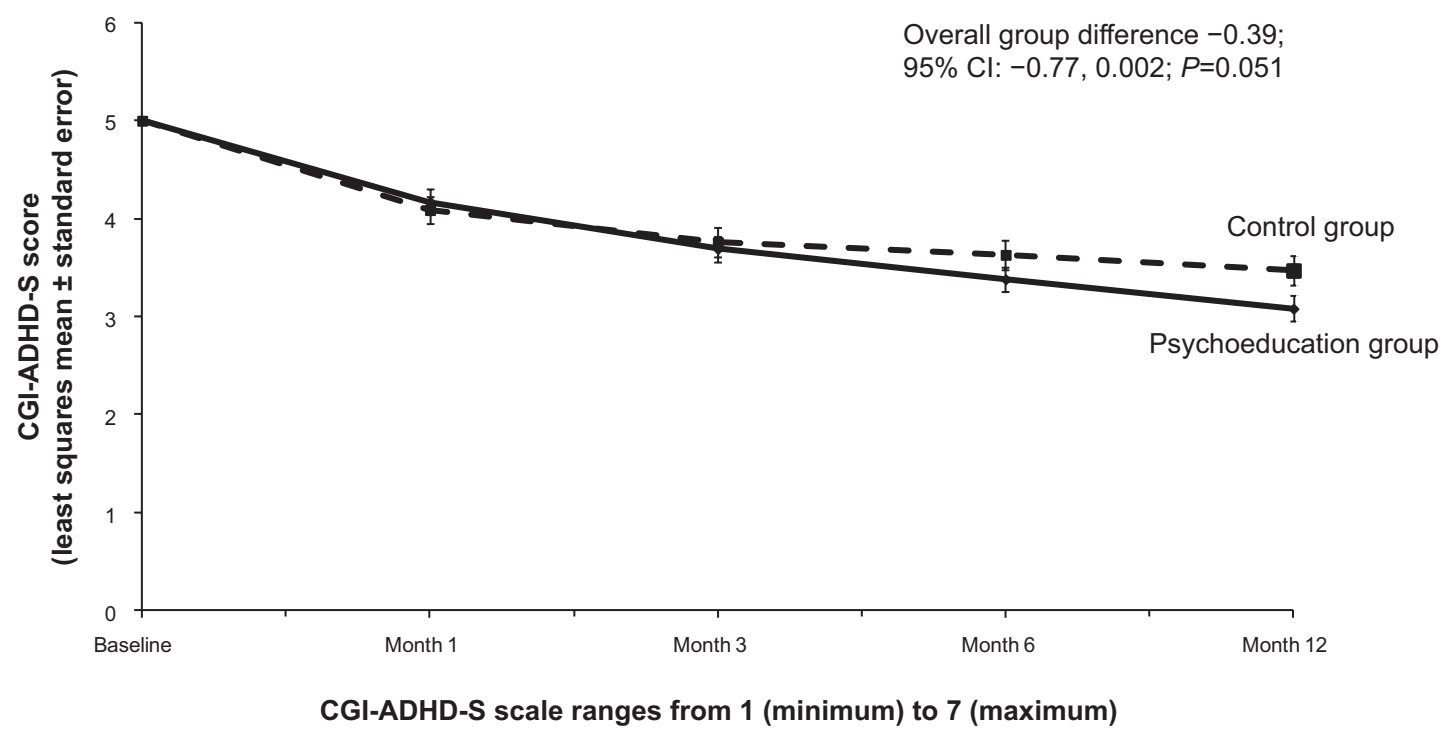

Figure 5 Change in CGI-ADHD-S total score (least squares mean estimate) for the psychoeducation and control groups.

Abbreviations: CGI-ADHD-S, Clinical Global Impression-Attention-Deficit/Hyperactivity Disorder-Severity scale; Cl, confidence interval. 


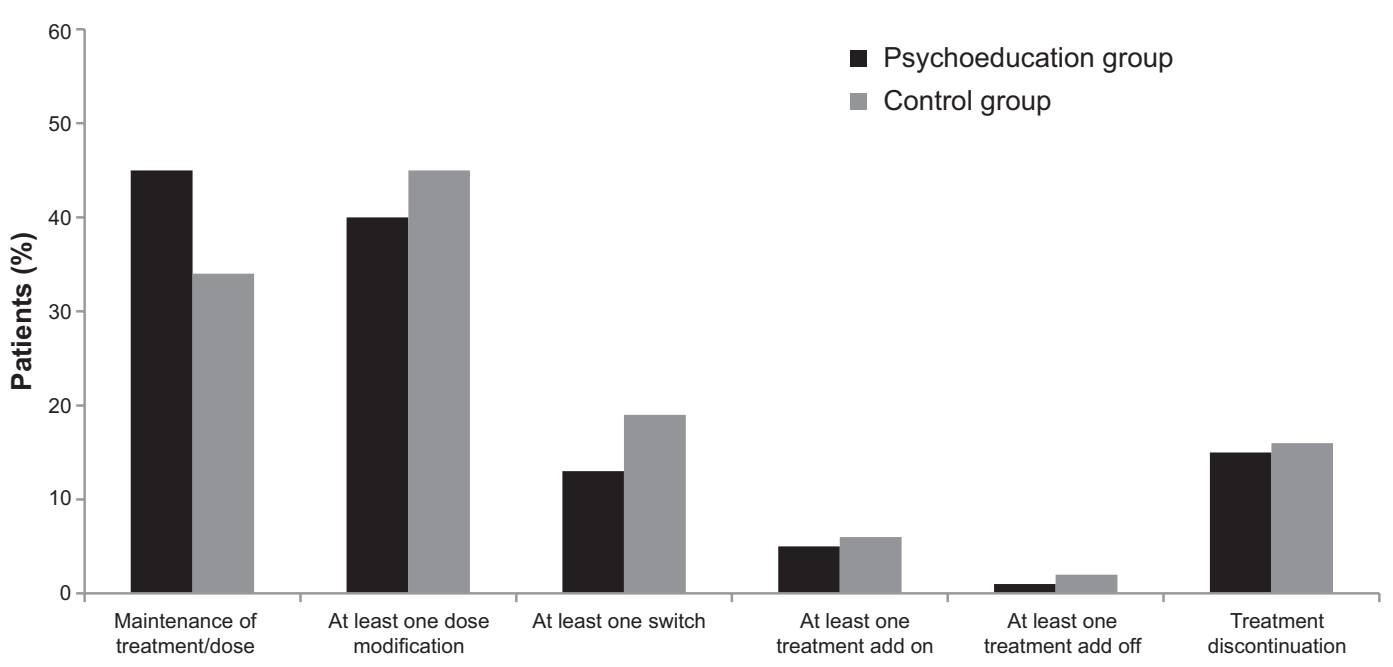

Figure 6 Drug usage patterns during the study

with stronger improvements seen in the psychoeducation group. However, no statistically significant differences in patient functioning were found between the groups using the WFIRS-P scale. Although the improvements in clinical symptoms in the psychoeducation group were modest, these may be important in some patients. The improvement in clinical symptoms may have been due partly to the fact that psychoeducation helped parents to recalibrate their feelings about the severity of their child's symptoms. In addition, it is possible that the beneficial effects of psychoeducation were time-related, with early effects being driven by medications ${ }^{28}$ but longer-term gains being achieved through an additive or interactive effect of medications and psychoeducation. It is also possible that ADHD medications and psychoeducation essentially served as a combined treatment approach, and that the psychoeducation program had a positive effect without influencing medication persistence. For example, it could have improved parenting self-efficacy, provided social support and advice on parenting practices, and improved parental understanding of ADHD, all of which could improve the child's functioning without influencing medication persistence. Regardless of the reason for the observed improvement in clinical symptoms, the results of the present study suggest that further investigations are warranted, particularly those evaluating the effects of psychoeducation on adherence and/or persistence and other outcomes in the longer term.

Limitations of this study included the slow recruitment process and eventual cessation of recruitment, leading to premature termination of the investigation before the planned sample size was reached. The complexity of the study design and the requirement for a large study population to achieve adequate statistical power were clear confounding factors in under-recruitment and may have contributed to the failure of the study to meet its primary endpoint. Reflecting this, the study was conducted over a prolonged period of almost 2.5 years. For some patients, it is also possible that the 12-month follow-up period was too short to detect any effects of psychoeducation on medication persistence, given that there were several positive indicators (albeit on secondary endpoints) of the benefits of psychoeducation. In addition, the study was not blinded; therefore, the possibility of investigator and informant (parent) assessment bias cannot be discounted. Finally, data on adherence with

Table 5 Protocol-emergent adverse events reported by $\geq 5 \%$ of patients

\begin{tabular}{llll}
\hline Parameter, $\mathbf{n}(\%)$ & $\begin{array}{l}\text { Psychoeducation } \\
(\mathbf{n}=\mathbf{1 4 4})\end{array}$ & $\begin{array}{l}\text { Control } \\
(\mathbf{n}=\mathbf{I 2 6})\end{array}$ & $\begin{array}{l}\text { Overall } \\
(\mathbf{n}=\mathbf{2 7 0})\end{array}$ \\
\hline At least one protocol-emergent adverse event & $54(37.5)$ & $60(47.6)$ & $114(42.2)$ \\
Decreased appetite & $21(14.6)$ & $22(17.5)$ & $43(15.9)$ \\
Headache & $20(13.9)$ & $10(7.9)$ & $30(11.1)$ \\
Abdominal pain & $13(9.0)$ & $12(9.5)$ & $25(9.3)$ \\
Pyrexia & $8(5.6)$ & $9(7.1)$ & $17(6.3)$ \\
Insomnia & $7(4.9)$ & $9(7.1)$ & $16(5.9)$ \\
Nasopharyngitis & $3(2.1)$ & $11(8.7)$ & $14(5.2)$ \\
\hline
\end{tabular}


medication (the extent to which a patient acts in accordance with the prescribed dosing regimen, such as dose, timing, and frequency) were not collected in this study, only data on medication persistence. Likewise, data on mechanistic variables that might explain the usefulness of psychoeducation, such as parental attitudes towards ADHD medication and the child's perception of their medication, were not collected.

\section{Conclusion}

This study did not demonstrate a significant advantage for parental psychoeducation plus medication over medication alone on time to withdrawal of medication when administered to parents of children undergoing treatment for newly diagnosed ADHD over a 12-month time period. However, patients with learning difficulties were more likely to discontinue treatment than those without such difficulties. Inconsistent but interesting effects of psychoeducation on other clinical outcomes were recorded and no safety issues were raised. These findings support the further evaluation of psychoeducation for parents of children being treated for ADHD in appropriately controlled trials.

\section{Acknowledgments}

The authors would like to thank the participating investigators and the patients and parents who contributed to this investigation. They would also like to thank Silvia Montaña and Laura Brun for their fundamental contribution in developing the psychoeducational program.

\section{Disclosure}

AM and PP are full-time employees of and shareholders in Eli Lilly. AH is a consultant for Eli Lilly, and a consultant and speaker for Shire. In the past 3 years, JF has participated in advisory activities, unrestricted educational activities, and research projects sponsored by Janssen, Eli Lilly, Shire, Roche, and public/not for profit agencies. EC has received compensation for serving as a consultant or speaker. Her institution has received research support or royalties from Eli Lilly, the Health Spanish Ministry Research Fund, the Ministry of Education Grant Research, Shire, and UCB. JQ has served as an investigator for Janssen-Cilag and Shire, and as a speaker for Janssen-Cilag, Shire, and Eli Lilly. RT was a member of the DSM-5 Work Group on ADHD and externalizing disorders, and has received speaker fees for an unrestricted talk, consultancy, and an advisory board meeting from Eli Lilly and Shire in the past 3 years. The authors acknowledge the editorial support provided by David Peters and Sue Chambers of Rx Communications, Mold, UK, which was funded by Eli Lilly and Co.

\section{References}

1. American Psychiatric Association. Diagnostic and Statistical Manual of Mental Disorders. 5th ed. Arlington, VA, USA: American Psychiatric Association; 2013.

2. Polanczyk G, de Lima MS, Horta BL, Biederman J, Rohde LA. The worldwide prevalence of ADHD: a systematic review and metaregression analysis. Am J Psychiatry. 2007;164:942-948.

3. Harpin V, Mazzone L, Raynaud JP, Kahle J, Hodgkins P. Long-term outcomes of ADHD: a systematic review of self-esteem and social function. $J$ Atten Disord. May 22, 2013. [Epub ahead of print].

4. Biederman J, Petty CR, Woodworth KY, Lomedico A, Hyder LL. Adult outcome of attention-deficit/hyperactivity disorder: a controlled 16-year follow-up study. J Clin Psychiatry. 2012;73:941-950.

5. Barbaresi WJ, Colligan RC, Weaver AL, Voigt RG, Killan JM, Katusic SK. Mortality, ADHD, and psychosocial adversity in adults with childhood ADHD. Pediatrics. 2013;131:637-644.

6. Esposito M, Gallai B, Parisi L, et al. Visuomotor competencies and primary monosymptomatic nocturnal enuresis in prepubertal aged children. Neuropsychiatr Dis Treat. 2013;9:921-926.

7. Cohen-Zion M, Anconi-Israel S. Sleep in children with attention-deficit hyperactivity disorder (ADHD): a review of naturalistic and stimulant intervention studies. Sleep Med Rev. 2004;8:379-402.

8. Esposito M, Antinolfi L, Gallai B, et al. Executive dysfunction in children affected by obstructive sleep apnea syndrome: an observational study. Neuropsychiatr Dis Treat. 2013;9:1087-1094.

9. Esposito M, Carotenuto M. Borderline intellectual functioning and sleep: the role of cyclic alternating pattern. Neurosci Lett. 2010;485: 89-93.

10. Biederman J, Faraone SV. Attention-deficit hyperactivity disorder. Lancet. 2005;366:237-248.

11. Pliszka S; for AACAP Working Group on Quality Issues. Practice parameters for the assessment and treatment of children and adolescents with attention-deficit/hyperactivity disorder. $J$ Am Acad Child Adolesc Psychiatry. 2007;46:894-921.

12. Canadian Attention Deficit Hyperactivity Disorder Resource Alliance. Practice Guidelines. Toronto, ON, Canada: Canadian Attention Deficit Hyperactivity Disorder Resource; 2010.

13. National Institute for Health and Care Excellence. Attention deficit hyperactivity disorder (CG72). Available from: http://www.nice.org. uk/CG072. Accessed January 23, 2014.

14. Montoya A, Colom F, Ferrin M. Is psychoeducation for parents and teachers of children and adolescents with ADHD efficacious? A systematic literature review. Eur Psychiatry. 2011;26:166-175.

15. Demidovich M, Kolko DJ, Bukstein OG, Hart J. Medication refusal in children with oppositional defiant disorder or conduct disorder and co-morbid attention-deficit/hyperactivity disorder: medical history and clinical correlates. J Child Adolesc Psychopharmacol. 2011;21: $57-66$.

16. Cramer JA, Roy A, Burrell A, et al. Medication compliance and persistence: terminology and definitions. Value Health. 2008;11: 44-47.

17. Barner JC, Khoza S, Oladapo A. ADHD medication use, adherence, persistence and cost among Texas Medicaid children. Curr Med Res Opin. 2011;27 Suppl 2:13-22.

18. Lawson KA, Johnsrud M, Hodgkins P, Sasane R, Crismon ML. Utilization patterns of stimulants in ADHD in the Medicaid population: a retrospective analysis of data from the Texas Medicaid program. Clin Ther. 2012;34:944-956.

19. Lachaine J, Beauchemin C, Sasane R, Hodgkins PS. Treatment patterns, adherence, and persistence in ADHD: a Canadian perspective. Postgrad Med. 2012;124:139-148. 
20. Faber A, Kalverdijk LJ, de Jong-van den Berg LTW, Hugtenburg JG, Minderaa RB, Tobi H. Parents report on stimulant-treated children in the Netherlands: initiation of treatment and follow-up care. $J$ Child Adolesc Psychopharmacol. 2006;16:432-440.

21. Coletti DJ, Pappadopulos E, Katsiotas NJ, Berest A, Jensen PS, Kafantaris V. Parent perspectives on the decision to initiate medication treatment of attention-deficit/hyperactivity disorder. J Child Adolesc Psychopharmacol. 2012;22:226-237.

22. Chan PW, DeBruyne JA. Parental concern towards the use of inhaled therapy in children with chronic asthma. Pediatr Int. 2000; 42:547-551.

23. Steinhoff KW. Attention-deficit/hyperactivity disorder: medication treatment-dosing and duration of action. Am J Manag Care. 2004; 10(4 Suppl):S99-S106.

24. Svanborg P, Therlund G, Gustafsson PA, Hägglöf B, Poole L, Kadesjö B. Efficacy and safety of atomoxetine as add-on to psychoeducation in the treatment of attention deficit/hyperactivity disorder. Eur Child Adolesc Psychiatry. 2009;18:240-249.

25. Rummel-Kluge C, Kluge M, Kissling W. Frequency and relevance of psychoeducation in psychiatric diagnoses: results of two surveys five years apart in German-speaking European countries. BMC Psychiatry. 2013;13:170.

26. Chuang JH, Hripcsak G, Heitjan DF. Design and analysis of controlled trials in naturally clustered environments: implications for medical informatics. J Am Med Inform Assoc. 2002;9:230-238.

27. Barkley RA. Psychosocial treatments for attention-deficit/hyperactivity disorder in children. J Clin Psychiatry. 2002;63 Suppl 12:36-43.

28. National Institute for Health and Care Excellence. Methylphenidate, atomoxetine and dexamfetamine for attention deficit hyperactivity disorder (ADHD) in children and adolescents. Available from: http://www.nice.org.uk/nicemedia/pdf/ta098guidance.pdf. Accessed January 23, 2014.

29. DuPaul GJ, Power TJ, Anastopoulos AD, Reid R. ADHD Rating Scale-IV. Checklist, Norms, and Clinical Interpretations. New York, NY, USA: Guilford Press; 1998.

30. Guy W. ECDEU Assessment Manual for Psychopharmacology, Revised. Bethesda, MD, USA: US Department of Health, Education, and Welfare; 1976.

31. Weiss MD, Weiss JR. A guide to the treatment of adults with ADHD $J$ Clin Psychiatry. 2004;65 Suppl 3:27-37.

32. Quintana H, Kelsey DK, Cherlin EA, et al. Transition from psychostimulants to atomoxetine in pediatric and adolescent patients with attention-deficit/hyperactivity disorder. Abstract P8.061 presented at the 18th European College of Neuropsychopharmacology Congress, October 22-26, 2005 Amsterdam, The Netherlands.
33. Dickson RA, Lee B, Turgay A, et al. Change in ADHD symptoms during 1 year of atomoxetine treatment. Presented at the 57th Annual Conference of the Canadian Psychiatric Association, Novmber 15-18, 2007, Montreal, QC, Canada.

34. Xie T, Waksman J. Design and sample size estimation in clinical trials with clustered survival times as the primary endpoint. Stat Med. 2003;22:2835-2846.

35. Campbell MK, Elbourne D, Altman D. CONSORT statement: extension to cluster randomised trials. BMJ. 2004;328:702-708.

36. Kaplan EL, Meier P. Nonparametric estimation from incomplete observations. J Am Stat Assoc. 1958;53:457-481.

37. Lin DY. Cox regression analysis of multivariate failure time data: the marginal approach. Stat Med. 1994;13:2233-2247.

38. Alder LD, Nierenberg AA. Review of medication adherence in children and adults with ADHD. Postgrad Med. 2010;122:184-191.

39. Hodgkins P, Sasané R, Christensen L, Harley C, Liu F. Treatment outcomes with methylphenidate formulations among patients with ADHD: retrospective analysis of a managed care population. Curr Med Res Opin. 2011;27 Suppl 2:53-62.

40. Marcus SC, Durkin M. Stimulant adherence and academic performance in urban youth with attention-deficit/hyperactivity disorder. $J$ Am Acad Child Adolesc Psychiatry. 2011;50:480-489.

41. Caisley H, Müller U. Adherence to medication in adults with attention deficit hyperactivity disorder and pro re nata dosing of psychostimulants: a systematic review. Eur Psychiatry. 2012;27:343-349.

42. Hugtenburg JG, Witte I, Heerdink ER. Determinants of compliance with methylphenidate therapy in children. Acta Paediatr. 2006;95:1674-1676.

43. Toomey SL, Sox CM, Finkelstein JA. Why do children with ADHD discontinue treatment? Clin Pediatr (Phila). 2012;51:763-769.

44. Kooij JJ, Rőssler M, Philipsen A, et al. Predictors and impact of non-adherence in adults with attention-deficit/hyperactivity disorder receiving OROS methylphenidate: results from a randomized placebocontrolled trial. BMC Psychiatry. 2013;13:36.

45. Takon L. Clinical use of a modified release methylphenidate in the treatment of childhood attention deficit hyperactivity disorder. Ann Gen Psychiatry. 2011;10:25.

46. Thyiruchelvam D, Charach A, Schachar RJ. Moderators and mediators of long-term adherence to stimulant treatment in children with ADHD. J Am Acad Child Adolesc Psychiatry. 2001;40:922-928.

47. Atzori P, Usala T, Carucci S, Danjou F, Zuddas A. Predictive factors for persistent use and compliance of immediate-release methylphenidate: a 36-month naturalistic study. J Child Adolesc Psychopharmacol. 2009;19:673-681.
Neuropsychiatric Disease and Treatment

\section{Publish your work in this journal}

Neuropsychiatric Disease and Treatment is an international, peerreviewed journal of clinical therapeutics and pharmacology focusing on concise rapid reporting of clinical or pre-clinical studies on a range of neuropsychiatric and neurological disorders. This journal is indexed on PubMed Central, the 'PsycINFO' database and CAS,

\section{Dovepress}

and is the official journal of The International Neuropsychiatric Association (INA). The manuscript management system is completely online and includes a very quick and fair peer-review system, which is all easy to use. Visit http://www.dovepress.com/testimonials.php to read real quotes from published authors. 